\title{
Application of Laney control chart in assessment of microbiological quality of oral pharmaceutical filterable products
}

\author{
M. E. Eissa \\ HIKMA Pharma, 2nd Industrial Zone, $6^{\text {th }}$ of October City, Giza, Egypt
}

\begin{abstract}
Evaluation of microbiological quality of pharmaceutical product is an important criterion for safe release of the dosage form to the drug market. Monitoring of the stability of monitored characteristic attribute is crucial to judge the degree of compliance of the manufacturing processes to reproducible procedures and good manufacturing practice (GMP). Two non-sterile liquid oral products were monitored during ten months of study for their microbiological stability characteristics using control charts. Since total viable count (TVC) results did not follow any distribution type, the application of Laney U' chart was found to be the best approach in such instances using relevant statistical software packages. The results of TVC for both products failed to follow the closest distribution in distribution fitting test at $a=0.05$. Interestingly, both products demonstrated several spots of out-of-control (OOC) states, although none of their batches showed out-of-specification (OOS) results. These OOC conditions require further investigations in order to provide corrective/preventive actions for improvement of bioburden stability of the manufactured pharmaceuticals. Accordingly, Laney attribute control chart may be regarded as very handy tool to evaluate microbiological characteristic of pharmaceutical dosage form, when results show significant over-dispersion or under-dispersion pattern in data distribution.
\end{abstract}

Keywords: Good manufacturing practice; Total viable count; Laney U' chart; Out-of-specification; Microbiological stability; Software packages

\section{Introduction}

Bioburden quality of pharmaceutical products and medicinal items is very important inspection characteristic that should be monitored regularly to ensure microbiological safety of the dosage forms for human consumption (WHO, 2007). Importantly, liquid drugs are specially products that at high risk from microbial deterioration and spoilage due to significant water activity $\left(\mathrm{a}_{\mathrm{w}}\right)$ of 0.6 or more (Bairdand Bloomfield, 1996).

There are many reported cases of microbial contamination of pharmaceutical products which required their withdrawal from the drug market (Coker, 2005; Clontz, 2009). The situation is exacerbated by incidences that emerged from consumption of dosage forms that are intruded by microflora (Kallings et al., 1996; Mwambete et al., 2009). Multiplication and proliferation of viable microbial cells in the product matrix may lead to catastrophic consequences if consumed by patients with several health complication problems may be encountered, in addition to adverse changes in product characteristics (Shaikh et al., 1988; Denyer and Baird, 1990).
Application of Shewhart charts has been used for decades in monitoring industrial processes. Quality control charts are very important tools in Six Sigma road map viz. Define, measure, analyze, improve and control (DMAIC). They are specifically important in the MAIC steps. However, drawing meaningful conclusion is largely dependent on proper selection of the best type of chart that fits selected data. There are broadly two types of control charts based on the type of collected results which are variable or attribute in nature (Eissa, 2016).

Due to the above described challenges in pharmaceutical industry, a trending system was sought for to establish an effective monitoring of two newly formulated and manufactured pharmaceutical oral liquid syrups. Furthermore, the system should be able not only to detect microbiological quality but also its stability which is reflection to the reproducibility of the manufacturing environment, materials and/or good manufacturing practice (GMP). In the same line, if the inspected characteristics of the manufactured products were stable then their stability will allow for prediction of their future properties. 


\section{Materials and methods}

The samples tested for this study includes two types of syrups namely Calcium/Vitamin D supplement (denoted by: OTTS) and the other is cough syrup (denoted by: TSAN). filled in dark amber glass bottles with plastic closures.All the samples were obtained from Class $\mathrm{C}$ production area of production facility in the industrial Zone, $6^{\text {th }}$ of October City, Giza, Egypt.

During a period of ten months, all batches produced from both products were subjected to quality control testing including microbiological monitoring (Khanom et al., 2013) 91 and 116 batches from OTTS and TSAN, respectively. Tests included total viable count (TVC) and pathogens testing as per compendial guideline (USP<61>, 2015; USP $<62>$, 2015).All reagents, materials, culture media and instruments were used as indicated by (Eissa and Mahmoud, 2015). Application of statistical software packages were used as detailed by (Eissa et al., 2015). Sampling scheme includes withdrawal of containers from the beginning (B), the middle $(\mathrm{M})$ and the end $(\mathrm{E})$ of the manufacturing process of the batch to cover the whole production period of the product. The pooled sample from representative containers (B, M and E) was tested microbiologically.

\section{Results and discussion}

Microbiological testing of specified pathogens (Staphylococcus aureus, Pseudomonas aeruginosa, bile-tolerant Gram-negative bacteria, Escherichia coli, Salmonella spp. and Burkholderiacepacia) for both products showed absence of any pathogenic and/or objectionable microbes in tested batches during the period of testing. The first five microorganisms are pharmacopeial microorganisms, while the last one is objectionable microbe that was subjected to routine monitoring after its detection in the water system. None of the tested batches demonstrated bioburden count above $100 \mathrm{CFU} / \mathrm{ml}$. TVC of all batches was below 70 $\mathrm{CFU} / \mathrm{ml}$, i.e. all batches of the two products met the acceptance criteria of the reference guideline. Gathered data did not follow normal distribution after both square root and logarithmic transformation as was suggested by Sandle (2004), even after removing the outliers from the original data (GraphPad Statistics Guide, 2016). Examination of the distribution pattern yielded Figure 1 that showed the difference in frequency distribution between both hypothetical and observed distribution frequencies of both liquid products. Distribution fitting study demonstrated that the results did not follow any type of distribution at $\mathrm{P}=0.05$. However, the closest distributions to OTTS and TSAN were binomial and Weibull (3), respectively. Nevertheless, they failed to follow the dedicated distributions at $a=0.05$. The gap between the ideal and the actual distribution profile of data could be demonstrated by histograms in Figure 2 . Moreover, outlier was evident with TSAN and the spreading of its result is higher than that of OTTS. The trend of microbial count showed that bioburden is centered around zero and one CFU/ml with TSAN and OTTS, respectively. Interestingly, Weibull is a continuous-type of probability distribution while microbial count is discrete in nature (CHOLAYUDTH, 2007; MathWave Technologies, 2016). Thus, this type of distribution in contrast to binomial distribution cannot be used to represent microbial population distribution (Weisstein, 2016). Figure 3 shows cumulative distribution function (CDF) for both syrups and their deviation from the supposed closest theoretical distribution. Based on descriptive statistics of the intervals, approximately $97 \%$ of the batches showed $\leq 1 \mathrm{CFU} / \mathrm{ml}$ with OTTS. Whereas about $95 \%$ of manufactured lots of TSAN showed $\leq 7$ $\mathrm{CFU} / \mathrm{ml}$.

The commonly used control chart in monitoring microbiological count in pharmaceutical industry is C-type when subgroup sizes are equal between samples (CHOLAYUDTH, 2007) and U-type when samples are not equal in size. Thus, $\mathrm{C}$ chart can be considered as a special case of $U$ chart (Wiesenfelder and McDonough, 2013). However, attribute control charts require that the underlying distribution to follow special pattern such as geometric, Poisson or binomial. Since the bioburden of both products did not follow any of these type of distribution, the best approach of using attribute chart was achieved by applying Laney modification to correct over-dispersion and/or under-dispersion (Laney, 2002; Minitab 17, 2016; Laney, 2016). Fig. 4 and 5 showed microbiological quality and its stability through successive industrial manufacturing process. Since microbiological specification control is one sided limit i.e. not more than (NMT) $100 \mathrm{CFU} / \mathrm{ml}$ (USP $<61>$, 2015). So, only upper values above mean value (U) were investigated. Accordingly, for OTTS there was observable shift in in the process mean successively, followed by random batches showing out-of-control (OOC) microbial count, most probably due to extraneous factors. On the other hand, TSAN batches showed randomly scattered pattern of OOC that might be attributed to assignable-causes-variation. Thus, although TVC was low in most cases yet an underlying microbiologically unstable manufacturing process between batches could be detected which requires further thorough investigation to elucidate the cause(s) for such difference in bioburden quality between product batches. Nevertheless, the currently used statistical process control (SPC) methodology provided valuable tool for monitoring microbiological quality and its trend during medicinal dosage 

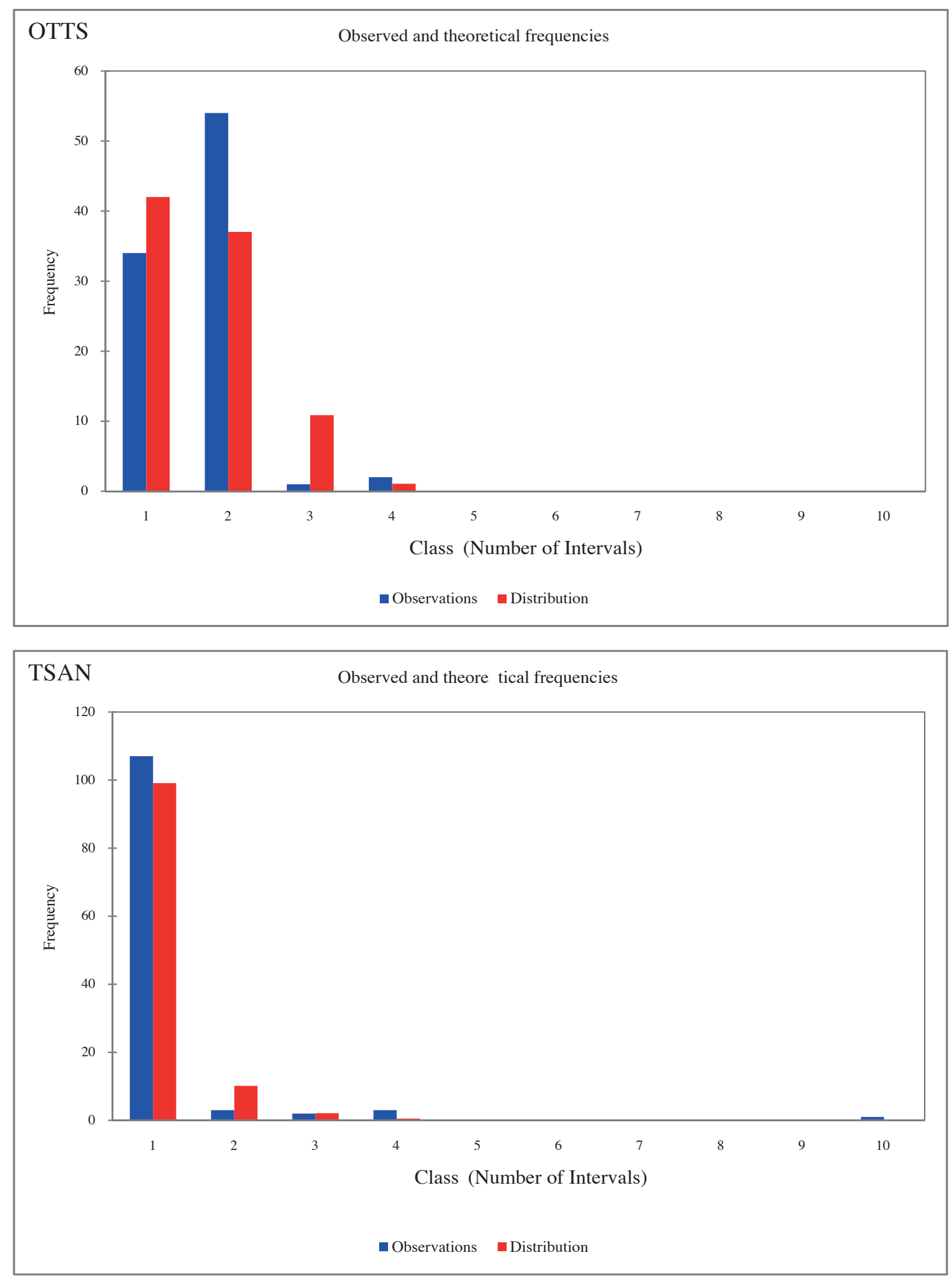

Fig. 1. Observed and expected distribution frequencies in microbial count of two liquid oral products: Calcium/Vitamin D supplement (OTTS) and cough (TSAN) syrups 

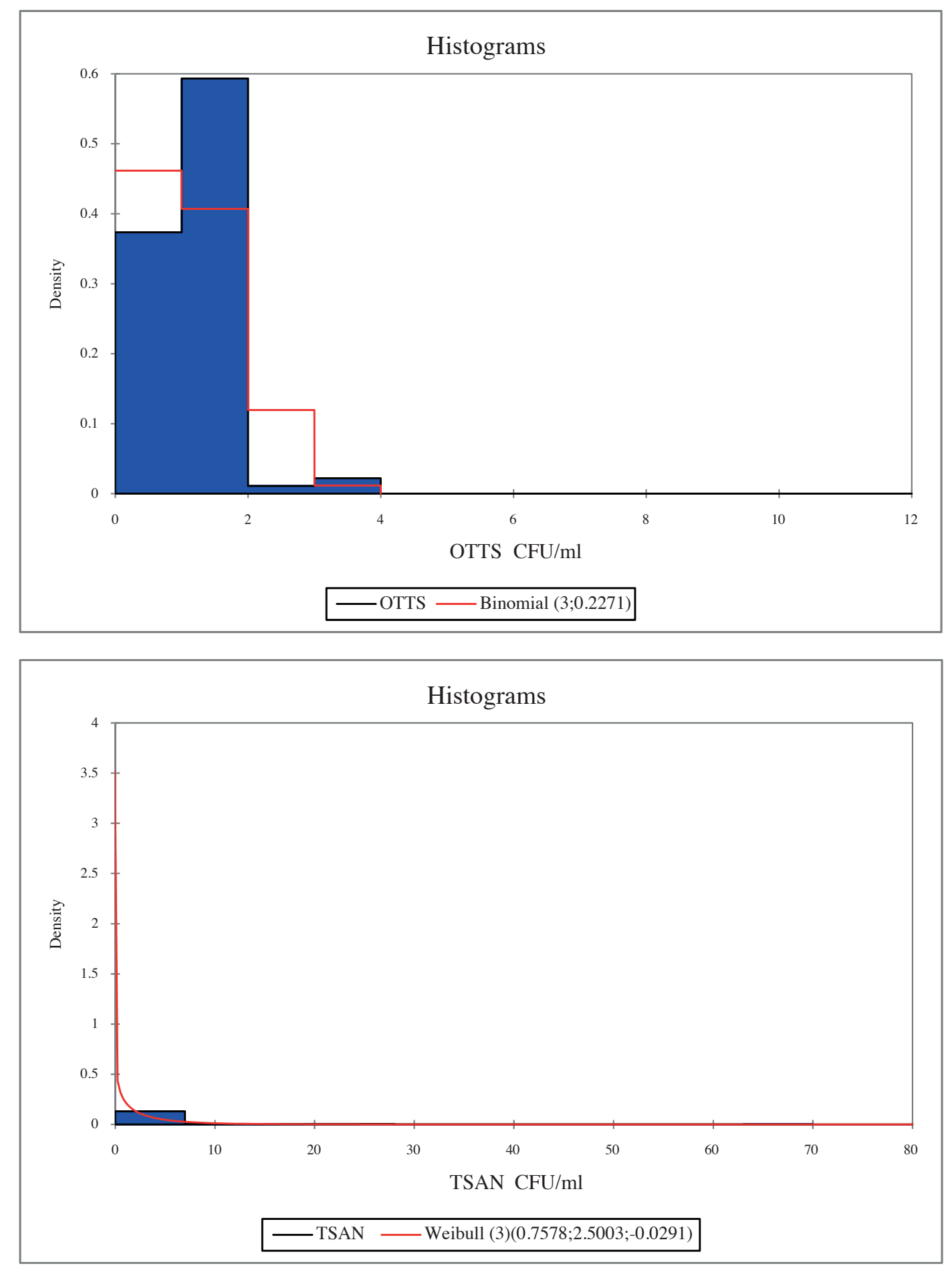

Fig. 2. Observed and expected histograms for two liquid oral products: Calcium/Vitamin D supplement (OTTS) and cough (TSAN) syrups, showing the difference between the actual microbial count distribution and the closest theoretical distribution 

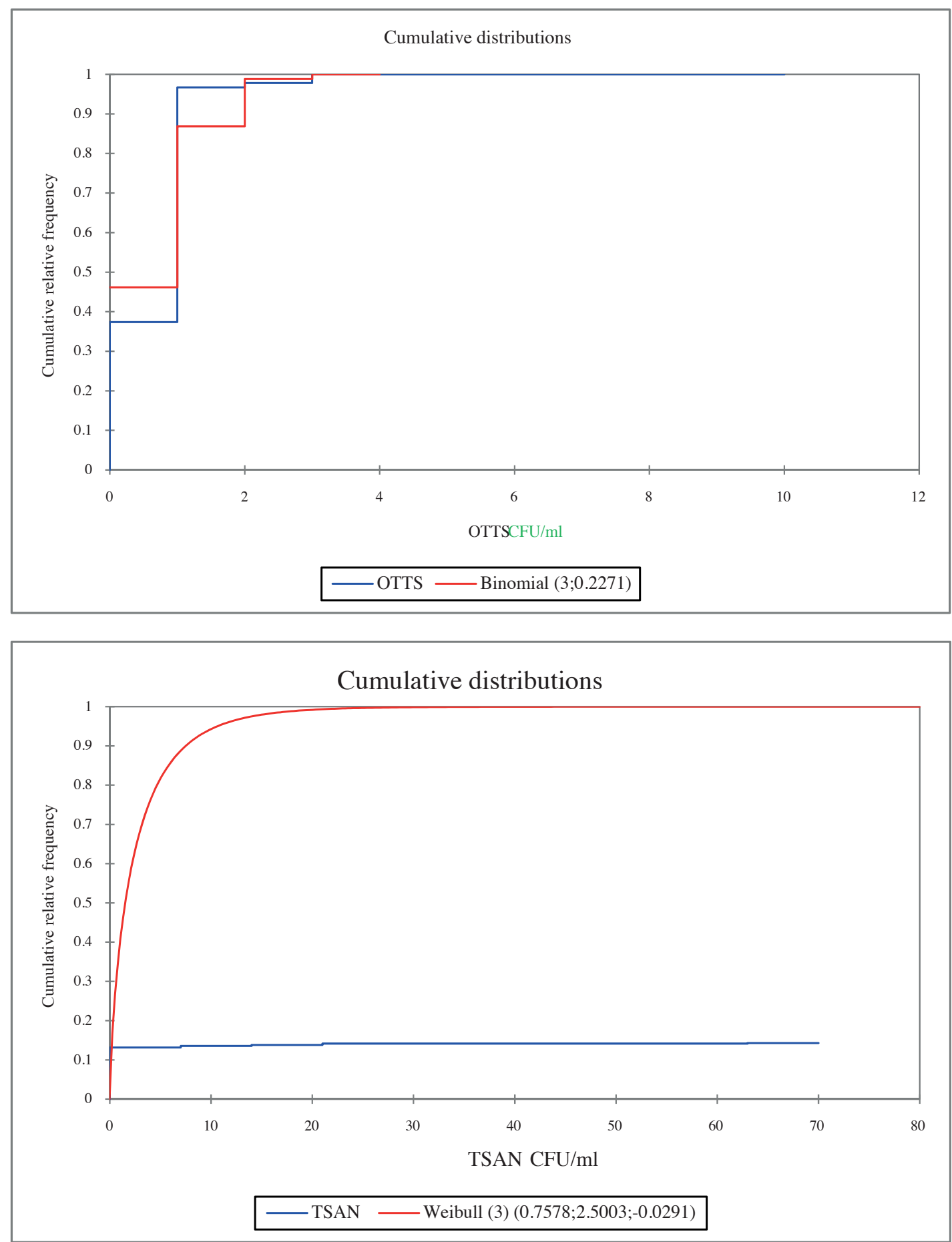

Fig. 3. Observed and expected cumulative relative frequency distributions for two liquid oral products: Calcium/Vitamin D supplement (OTTS) and cough (TSAN) syrups, showing the difference between the actual and the closest theoretical microbial count distribution 


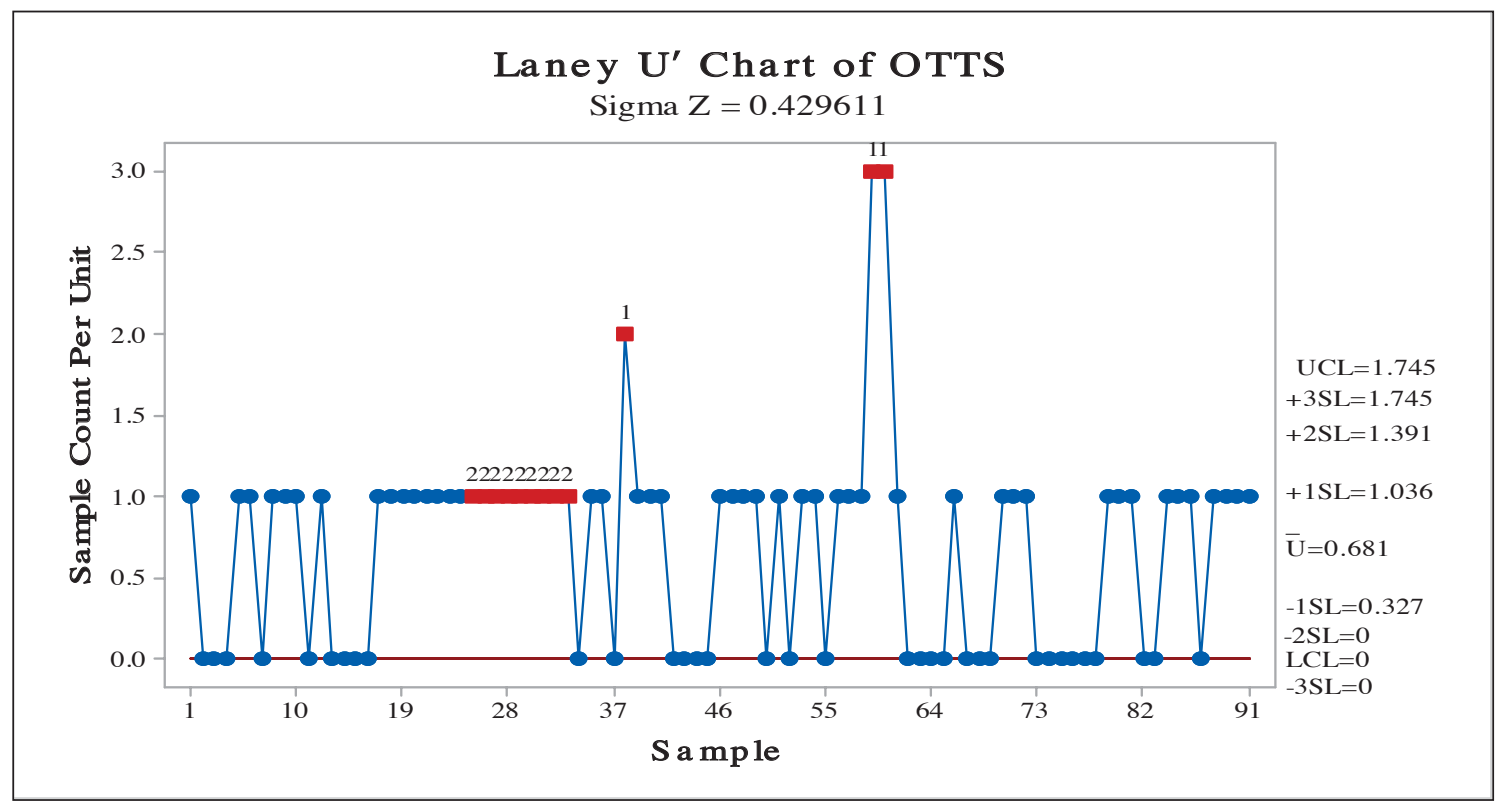

Fig. 4. Laney attribute control chart for bioburden in Calcium/Vitamin D supplement (OTTS) syrup in chronological order of the manufactured batches showing out-of-control values $(1=$ Abnormal result due to extraneous factor and $2=$ Drift in the process average)

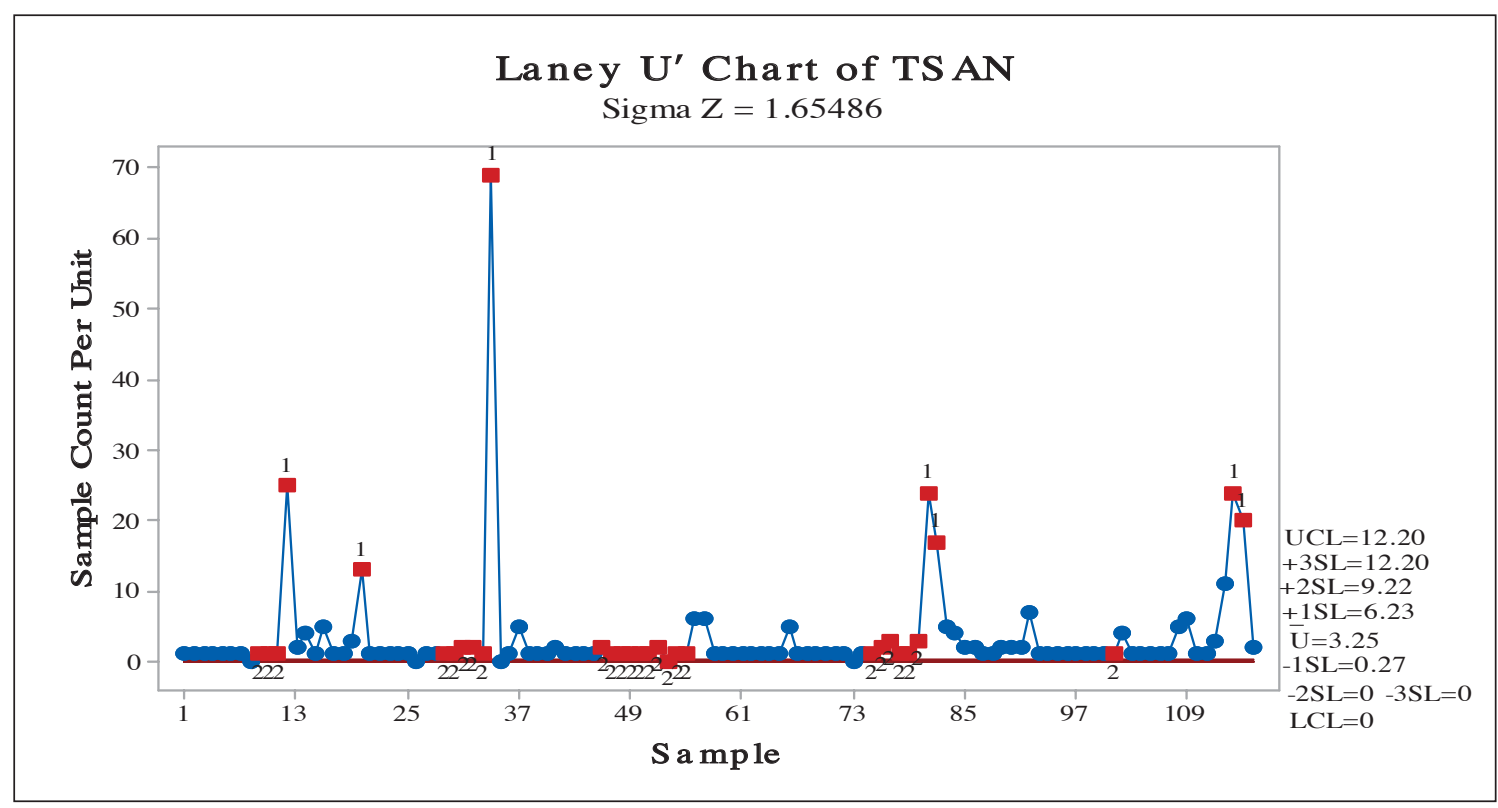

Fig. 5. Laney attribute control chart for cough (SCAN) syrup in chronological order of the manufactured batches showing out-of-control values. $(1$ = Abnormal result due to extraneous factor and 2 = Drift in the process average) 
forms manufacturing in pharmaceutical plant. In the current case, control charts revealed non-consistent microfloral quality between manufactured batches, although none of them exceeded specification limit of TVC.

Several factors should be investigated for fluctuation of the contamination such as consistency of the microbial quality for purified water, raw materials (active and excipients), primary packaging components, manufacturing environment and GMP (Gad et al., 2011). Since both products contains preservatives, the masking of the actual bioburden problem may be intensified if coupled with condition of viable-but-not-culturable (VBNC) state of microbial cells (Watkins and Jian, 1997). This problem has been specifically addressed with conventional culture media. In such instances, control charts may provide early warning signal for the hidden problem that may appear inthe future, even if the products are apparently meeting the specification.

\section{Conclusion}

Process control monitoring provides indispensable tool for evaluation of the quality characteristic in quality control monitoring. Regular monitoring tests that meet acceptance criteria for tested products alone cannot be used alone as sole measure to elucidate product quality with underlying manufacturing environment compliance. Data trending must follow result issuing to determine the stability of the measured quality attribute which may help in investigation of excursions that may emerge. Attribute control chart is suitable for microbiological count. However, in case of results that do not follow appropriate distributions, Laney-modified control chart may provide appropriate approach to monitor the quality characteristic attribute in such cases in attempt to deliver safe dosage forms to patients.

\section{References}

Baird R and Bloomfield S (1996), Microbial quality assurance in cosmetics, toiletries and non-sterile pharmaceuticals, Taylor \& Francis, London.

Cholayudth P (2007), Application of Poisson Distribution in Establishing Control Limits for Discrete Quality Attributes, http://www.ivtnetwork.com/sites /default/files/PoisionDistrib_01.pdf

Clontz L (2009), Microbial Limit and Bioburden Tests: Validation approaches and global requirements, CRC Press, Boca Raton.
Coker M (2005), An assessment of microbial contamination during drug manufacturing in Ibadan, Nigeria, Eur. J. Scientific Res. 7: 19-23.

Denyer Sand Baird R(1990), Guide to microbiological control in pharmaceuticals, $1^{\text {st }}$ Ed., Ellis Horwood, New York.

Eissa M (2016), Application of Control Charts in Quality Characteristics Evaluation of Microbiological Media. J. Adv. Res. Pharm. Sci. Pharmacol.Interv. 1(1\&2): $1-13$.

Eissa M and Mahmoud A (2015), Development of methods for microbial recovery: pharmaceutical dosage forms including drugs with antimicrobial properties (Study III), Eur. J. Pharm. Med. Res. 2(4): 537-549.

Eissa M, Seif Mand Fares M (2015), Assessment of purified water quality in pharmaceutical facility using six sigma tools, Int. J. Qual. Assur. 6(2): 54-72.

Gad G, Aly R, and Ashour M (2011), Microbial Evaluation of Some Non-sterile Pharmaceutical Preparations Commonly Used in the Egyptian Market, Tropical Journal Of Pharmaceutical Research 10(4): 437-445.

GraphPad Statistics Guide (2016), How to: Identify outliers, http://www.graphpad.com/guides/prism/7/statistics/in dex.htm?stat_how_to_removing_outliers.htm

Kallings LO, Ringertz O and Silverstolpe L (1996), Microbiological contamination of medical preparations, Acta Pharmaceutica Suecica 3(3): 219-228.

Khanom S, Das KK, Banik S and Noor R (2013), Microbiological analysis of liquid oral drugs available in Bangladesh, Int. J. Pharm. Pharma. Sci. 5(4): 479-482.

Laney D (2002), Improved Control Charts for Attributes, Quality Engineering, 14(4): 531-537.

Laney D (2016), P-Charts and U-Charts Work (But Only Sometimes), http://www.qualitydigest. com/ currentmag/ departments/what_works. shtml

Math Wave Technologies (2016), EasyFit - Weibull Distribution, http://www. mathwave. com/ help /easyfit/html/analyses/ distributions/weibull. html 
Minitab 17 (2016), Attribute Control Charts, http://support.minitab.com/en-us/minitab/17/Assistant _Attribute_Control_Charts.pdf

Mohammed M and Laney D (2006), Overdispersion in health care performance data: Laney's approach, Quality And Safety In Health Care, 15(5): 383-384.

Mwambete KD, Temu MJ and Fazleabbas FS (2009), Microbiological assessment of commercially available quinine syrup and water for injections in Dar Es Salaam, Tanzania. Tropical Journal of Pharmaceutical Research $\mathbf{8}(5)$.

ORA.007, Version 1.2 (2015), Pharmaceutical Microbiology ManualScienceresearch, http://www.fda. gov/downloads/ScienceResearch/Field Science/UCM397228.pdf

Sandle T (2004), An approach for the reporting of microbiological results from water systems, PDA J. Pharm Sci. Technol. 58(4): 231-237.

Shaikh D, Jamshed TA and Shaikh R (1988), Microbial contamination of pharmaceutical preparations, Pak. J. Pharm. Sci. 1: 61-6.

USP General Chapter <61> (2015), Microbiological Examination Of Non-sterile Products: Test For Specified Microorganisms, United States Pharmacopeia 38/National Formulary 33, Baltimore, MD, USA.

USP General Chapter <62> (2015), Microbiological Examination Of Nonsterile Products: Microbial Enumeration Tests, United States Pharmacopeia 38/National Formulary 33, Baltimore, MD, USA.
Watkins J and Jian X (1997), Cultural methods of detection for microorganisms: recent advances and successes.

Weisstein E (2016), Binomial Distribution - from Wolfram Math World, http://mathworld.wolfram.com/ BinomialDistribution.html

Wiesenfelder $\mathrm{H}$ and McDonough M (2013), Types of Attribute Control Charts: The P Chart, NP Chart, C Chart and U Chart, Brighthub Project Management, h t t p : / / w w w . b r i g h t h u b p m . c o m / six-sigma/63117-types-of-attribute-control-charts/

WHO, World Health Organization (2007), Quality assurance of pharmaceuticals: a compendium of guidelines and related materials, Good manufacturing practices and inspection (Vol. 2).

Received: 13 November 2016; Revised: 01 January 2017;

Accepted: 29 May 2017. 